\title{
Recurrent Xanthine Stone Formation in a Lesch-Nyhan Patient: A Case Report
}

\author{
Ari Bernstein ${ }^{\mathrm{a}, \mathrm{d}}$, Mark C. Liszewski ${ }^{\mathrm{b}}$, Beth Drzewiecki ${ }^{\mathrm{c}}$
}

\begin{abstract}
Lesch-Nyhan disease is a rare genetic condition that presents as a deficiency in the hypoxanthine-guanine phosphoribosyltransferase (HGPRT) enzyme of the purine nucleotide recycling pathway. $\mathrm{Pa}$ tients with Lesch-Nyhan commonly present with uric acid urolithiasis, but rare cases of xanthine stone formation have been reported. Treatment for these recurrent xanthine stones has proven difficult and there remains no standard protocol for this presentation. Impaired renal function is of critical concern with current treatment practices and outcomes. We report a case of a 3-year-old boy with Lesch-Nyhan disease who presented with recurrent obstructing xanthine stones.
\end{abstract}

Keywords: Lesch-Nyhan; Xanthine; Urolithiasis; HPRT; HGPRT; Stone

\section{Introduction}

Lesch-Nyhan disease is a rare, $\mathrm{X}$-linked, genetic condition caused by a mutation in the HPRT gene. Loss of HPRT function leads to excess uric acid production, often leading to uric acid crystals that can accumulate in joints and/or uric acid urolithiasis which may lead to urinary tract obstruction. Typical symptoms include excess uric acid production, self-injurious behavior, and developmental delay. The neurological symptoms are of unknown etiology $[1,2]$.

\section{Case Report}

A 3-year-old boy previously diagnosed with Lesch-Nyhan

Manuscript submitted June 22, 2017, accepted July 21, 2017

aAlbert Einstein College of Medicine, 1925 Eastchester Road Apt 23G, Bronx, NY 10461, USA

bDepartment of Radiology, Montefiore Medical Center, 111 East 210th Street, Bronx, NY 10467, USA

'Department of Urology, Montefiore Medical Center, Medical Arts Pavilion, 3400 Bainbridge Avenue, MMC-MAP, Room 5, Bronx, NY 10467, USA

${ }^{\mathrm{d} C o r r e s p o n d i n g ~ A u t h o r: ~ A r i ~ B e r n s t e i n, ~ A l b e r t ~ E i n s t e i n ~ C o l l e g e ~ o f ~ M e d i c i n e, ~}$ 1925 Eastchester Road Apt 23G, Bronx, NY 10461, USA.

Email: abernst2@mail.einstein.yu.edu disease presented with abdominal pain, nausea, and vomiting in September 2014. He had a similar presentation to the hospital 3 months prior and was noted to have right hydroureteronephrosis on renal sonogram at that time, which persisted on repeat sonogram. No urolithiasis was noted on sonogram. He underwent an abdominal/pelvic CT scan which revealed an obstructing stone in his right ureter of $7 \mathrm{~mm}$ (Fig. 1) and a large left renal pelvis stone of about $1 \mathrm{~cm}$ (Fig. 2). His renal function was elevated with a $\mathrm{Cr}$ of $0.7 \mathrm{mg} / \mathrm{dL}(0.4-0.7 \mathrm{mg} /$ $\mathrm{dL}$ ) and his eGFR was $59.9 \mathrm{~mL} / \mathrm{min} / 1.73 \mathrm{~m}^{2}$ calculated using the Schwarz equation. He was currently on treatment with allopurinol and his serum uric acid was $3.7 \mathrm{mg} / \mathrm{dL}(2.1-5.6$ $\mathrm{mg} / \mathrm{dL})$ and his urinary uric acid level was $8.8 \mathrm{mg} / \mathrm{dL}(9.0$ - $114 \mathrm{mg} / \mathrm{dL}$ ). Urine $\mathrm{pH}$ was 7.0. The patient underwent cystourethroscopy and bilateral lithotripsy along with bilateral ureteral stent placement. Stone analysis revealed $100 \%$ xanthine stones. The patient was discharged and sent home from this encounter but has since presented twice with obstructing xanthine stones that have required intervention over the past several years.

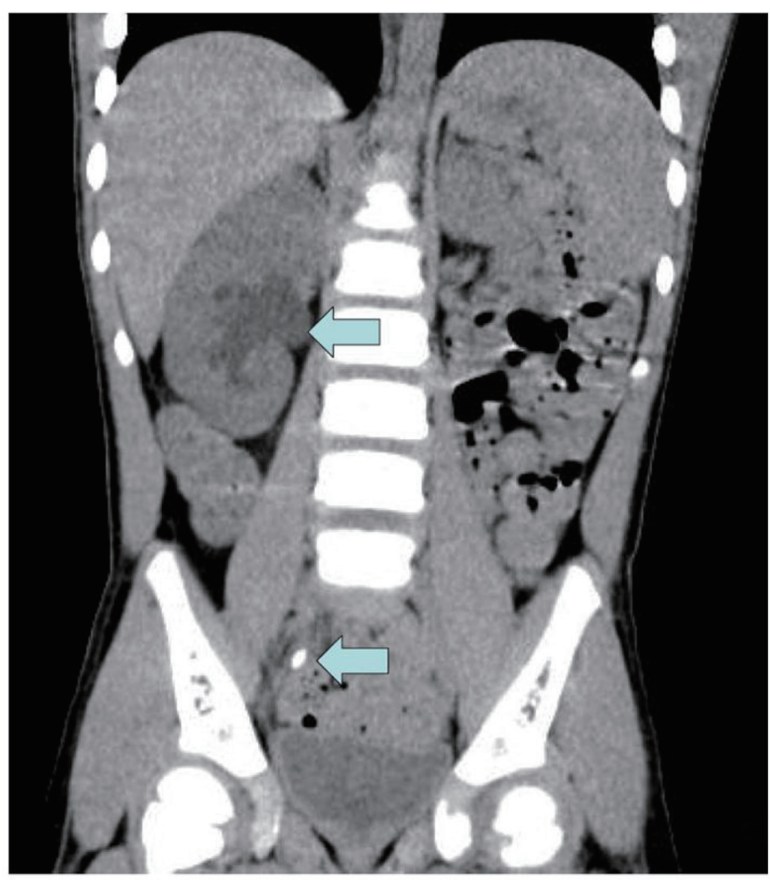

Figure 1. Right hydronephrosis and obstructing ureteral stone. 


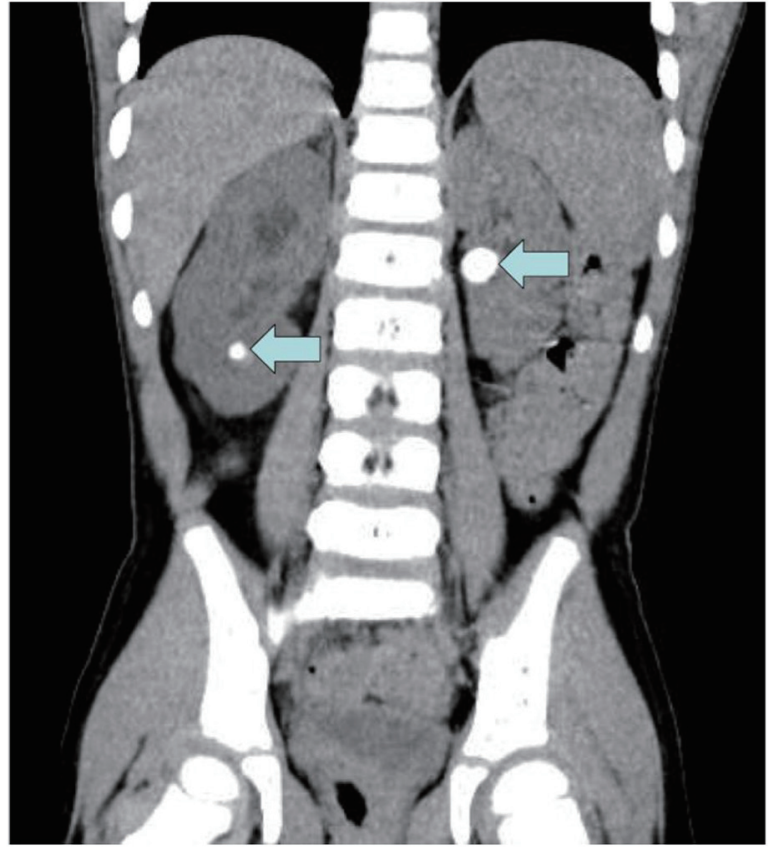

Figure 2. Left renal pelvis and right lower pole stones on CT.

\section{Discussion}

\section{Etiology of zanthine stones}

Lesch-Nyhan is an X-linked genetic disorder, primarily affecting males while women are often asymptomatic carriers. The normal HPRT gene codes for hypoxanthine-guanine phosphoribosyltransferase (HGPRT), an enzyme intimately involved in the recycling and regeneration of purine nucleotides. The HPRT mutation leads to a deficiency in HGPRT and thus both an inability to sufficiently recycle these nucleotides and an increase in uric acid production (Fig. 3). The HGPRT deficiency further leads to increased de novo synthesis of purines in an attempt to compensate for decreased recycling. This compensatory process results in an exacerbated effect of excess uric acid production, leading to hyperuricemia and hyperuricosuria [1-3].

Xanthine is a precursor to uric acid in the degradation of purines, and its conversion to uric acid is achieved by the enzymatic action of xanthine oxidase. Allopurinol, a xanthine oxidase inhibitor, and a commonly prescribed drug for patients with Lesch-Nyhan disease, works to decrease production of uric acid and thus prevent uric acid urolithiasis. Despite its success, allopurinol therapy has been considered a potential causative agent of xanthine stone formation by increasing xanthine levels in its inhibition of xanthine's conversion to uric acid [4]. Allopurinol typically has the added effect of decreased de novo purine synthesis, but in the case of HGPRT deficiency, this effect has been observed to be absent [1]. The uninhibited conversion of guanine to xanthine by guanine deaminase with allopurinol treatment may also play a role in the recurrence of xanthine stone formation. To our knowledge, guanine levels have not been studied in relation to xanthine crystalluria in patients with Lesch-Nyhan. A widely accepted mechanism of xanthine stone formation in these rare presentations of LeschNyhan disease has not yet been determined.

\section{Treatment and outcomes}

Treatment of his recurrent xanthine urolithiasis has included maximizing his daily allopurinol treatment with a multidisciplinary and inter-institutional team of a primary care physician, urologist, geneticist, nephrologist, gastroenterologist,

\section{DE NOVO SYNTHESIS}

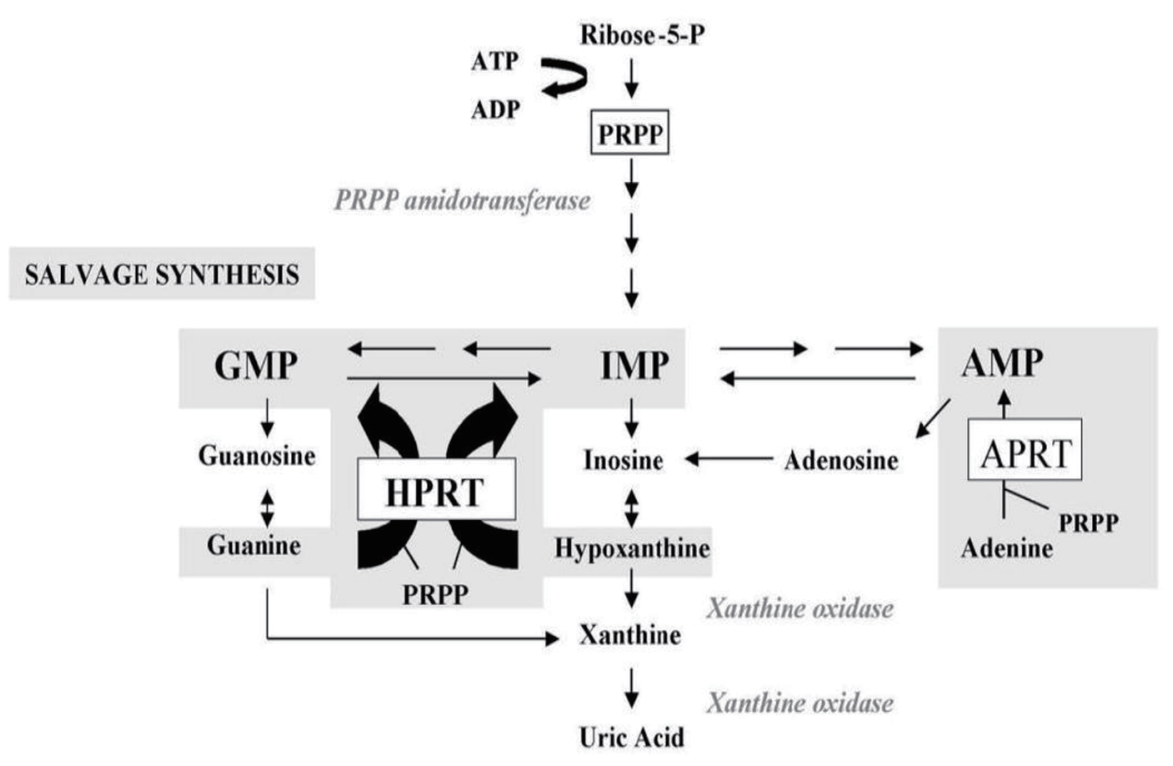

Figure 3. HGPRT enzyme function in purine metabolism. 
and neurologist, placement of a gastrostomy tube for adequate fluid hydration and urine output, alkalinization of urine with potassium citrate, and repeated cystoscopy and ureteroscopy with laser lithotripsy as needed for obstructing stones [5, 6]. Volpe et al observed that cessation of allopurinol therapy led to a significant reduction in xanthine stone recurrence in a LeschNyhan patient, but this approach increases the risk for uric acid stone formation [7]. Urinary xanthine and hypoxanthine levels have been followed and have significant variation between collections. Despite these measures he continues to have high levels of xanthine crystalluria and recurrent stone formation.

Xanthine urolithiasis is extremely rare, even for LeschNyhan patients with the HPRT mutation. There are many clinical challenges to treatment and prevention of the stones as they are radiolucent on radiography and can be difficult to evaluate on sonography due to crystalluria. Repeat CT scans are not recommended in children. The natural history of this disease is unclear, and prevention of long-term renal dysfunction is of critical concern. As we continue to closely monitor renal function in this patient, we may begin looking at urinary guanine levels as a predictor of recurrent xanthine stone formation risk, as guanine can still be converted into xanthine despite HPGRT deficiency and allopurinol inhibition of xanthine oxidase.

\section{Conflicts of Interest}

The authors have no conflicts of interest.

\section{References}

1. Ogawa A, Watanabe K, Minejima N. Renal xanthine stone in Lesch-Nyhan syndrome treated with allopurinol. Urology. 1985;26(1):56-58.

2. Lesch M, Nyhan WL. A familial disorder of uric acid metabolism and central nervous system function. Am J Med. 1964;36:561-570.

3. Torres RJ, Puig JG. Hypoxanthine-guanine phosophoribosyltransferase (HPRT) deficiency: Lesch-Nyhan syndrome. Orphanet J Rare Dis. 2007;2:48.

4. Torres RJ, Prior C, Puig JG. Efficacy and safety of allopurinol in patients with the Lesch-Nyhan syndrome and partial hypoxanthine- phosphoribosyltransferase deficiency: a follow-up study of 18 Spanish patients. Nucleosides Nucleotides Nucleic Acids. 2006;25(9-11):1077-1082.

5. Hiraishi K, Nakamura S, Yamamoto S, Kurokawa K. Prevention of xanthine stone formation by augmented dose of allopurinol in the Lesch-Nyhan syndrome. Br J Urol. 1987;59(4):362-363.

6. Rebentisch G, Stolz S, Muche J. [Xanthinuria with xanthine lithiasis in a patient with Lesch-Nyhan syndrome under allopurinol therapy]. Aktuelle Urol. 2004;35(3):215221.

7. Volpe P, Peyrottes A, Lammle M, Saquet D, Choquenet C. [Xanthine urinary calculus in a patient with Lesch-Nyhan syndrome. Apropos of a case]. Prog Urol. 1997;7(1):7477. 